\title{
Hippocampal-cerebellar involvement in enhancement of performance in word-based BRT with the presence of background noise: an initial fMRI study
}

\author{
Hanani Abdul Manan¹, Elizabeth A. Franz², Ahmad Nazlim Yusoff', Siti Zamratol-Mai Sarah \\ Mukari $^{1}$ \\ 1 - Universiti Kebangsaan Malaysia, Kuala Lampur, KL, Malaysia \\ 2 - University of Otago, Dunedin, OT, New Zealand
}

\begin{abstract}
Background noise may impose deleterious effects on cognitive processing. However, noise below the threshold level may increase the ability to detect stimuli via stochastic resonance mechanisms (SR). The present study investigates whether task performance is deteriorated or enhanced by $5-\mathrm{dB}$ SNR and, if the task performance is enhanced, whether this facilitation in performance points to a particular neural area that serves to attenuate noise and/or increase effective task performance. The areas of interest are the cerebellum and hippocampus due to their roles in working memory (WM) and their links with attention. Fifteen healthy young Malay adults performed three tasks during fMRI scanning: listening to babble noise (N), WM task in quiet (WMQ), and WM task in noise (WMN). Activated regions during N are bilateral STG and MTG. Both WM tasks produced similar activation in a network of areas in the frontal, temporal and parietal lobes. However, the two tasks demonstrated marked differences in the left hippocampus, right posterior cerebellum, and bilateral anterior cerebellum. Moreover, the results obtained from the behavioral task demonstrated that participants responded better in the presence of noise. These results support the hypothesis that the left hippocampus, right posterior cerebellum, and bilateral anterior cerebellum may be involved in attenuating noise and/or increasing attention to task performance, which could be due to SR mechanisms operating in the presence of noise. These results collectively suggest leftward asymmetries during the tasks with the right posterior cerebellum, bilateral anterior cerebellum, and left hippocampus providing compensatory attention processes, at least in the context of this study.
\end{abstract}

Keywords: fMRI; phonological working memory; cerebellum; hippocampus; stochastic resonance mechanism.

Received 21 May 2012; received in revised form 13 September 2012; accepted 24 October 2012. Available online 28 December 2012.

\section{Introduction}

Cognitive processing is easily disturbed by incompatible environmental stimulation, which distracts attention from the main tasks. This is assumed to be due to the competition for attentional resources between the distracting (noise) and the target stimuli (Soderlund et al., 2010). Noise is typically conceived as being detrimental to the complex cognitive performance and

Hanani Abdul Manan and Ahamd Nazlim Yusoff, Diagnostic Imaging and Radiotherapy Program, School of Diagnostic and Applied Health Sciences, Faculty of Health Sciences, Universiti Kebangsaan Malaysia. Elizabeth A. Franz, Department of Psychology and fMRIotago, University of Otago, New Zealand. Siti Zamratol-Mai Sarah Mukari, School of Rehabilitation Sciences, Faculty of Health Sciences, Universiti Kebangsaan Malaysia. Correspondence regarding this article should be directed to: Hanani Abdul Manan, School of Diagnostic and Applied Health Sciences, Faculty of Health Sciences, Universiti Kebangsaan Malaysia. Jalan Raja Muda Abdul Aziz, 50300. Kuala Lumpur, Malaysia. Phone: +603-26878084. Fax: +603-26878108. E-mail: hnani81@ gmail.com. the negative effects of noise on cognitive processing especially in attention, working memory (WM), and language have been established by many researchers (Dos Santos Sequeira, Specht, Hamalainen, \& Hugdahl, 2008; Kozou et al., 2005). It is also known that different types of background noise affect cognitive performance in different ways. For example, background noise in meaningful word-based contexts (e.g., babble noise and cocktail party noise) is more detrimental than other types of background noise (e.g., traffic, aircraft and highway) (Kujala \& Brattico, 2009; Sorqvist, 2010).

However, there are reports of contradictory findings where moderate amounts of auditory noise will improve the cognitive performance. A recent computational model is based on the concepts of stochastic resonance (SR), postulating that noise can enhance the detectability of an input signal (Moss, Ward, \& Sannita, 2004; Rousseau \& Chapeau-Blondeau, 2004; Yamamoto et al., 2002). SR is a proposed mechanism where the presence of random interference (moderate auditory noise) up to a finite level of intensity enhances sensitivity to external stimuli by increasing attention (Yamamoto et al., 2002). Enhancement 
to the detection level is postulated to be due to the system resonating at a particular noise level (Rousseau \& Chapeau-Blondeau, 2004). Previous studies have reported that the human central nervous system is able to utilize the noise-enhanced sensory information so that the sensitivity of sensory neurons on weak signals is optimized by adding noise (Yamamoto et al., 2002). However, noise beyond the optimal level may decrease the detectability of information or stimuli (Moss et al., 2004).

The present investigation focuses on the cerebellum and hippocampus due to their known associations with attention during cognitive processing (Alexander, Gillingham, Schweizer, \& Stuss, 2011; Marvel \& Desmond, 2010: Bledsoe, Semrud-Clikeman, \& Pliszka, 2009; TederSalejarvi, Pierce, Courchesne, \& Hillyard, 2005; Kujala et al., 2004; Gottwald, Mihajlovic, Wild, \& Mehdorn, 2003). In order to assess cognitive performance and interference of background noise in the present study, a word-based backward repeat span task (word-based BRT) was used. This task was adapted from Light \& Anderson (1985) and was designed to investigate WM capacity (Morra, 1994; Henry \& Millar, 1991). A word-based instead of digit-based task was chosen because of the known roles of speech processing in WM (Light \& Anderson, 1985; Baddeley, 1992, 2003a,b). The use of word-based stimuli also enabled us to standardize the number of syllables used, given that syllables tend to vary in number across words. This is important due to the fact that the performance of phonological WM may decrease string lengths exceeding a storage capacity limit (Baddeley, 2000; Baddeley, Allen, \& Vargha-Khadem, 2010).

As suggested earlier, the deleterious effects of noise on cognitive performance is assumed to be due to inability of individuals to discriminate between noise and target stimuli. The idea of a SR mechanism is relatively new in neurological science, especially in relation to the WM system. Moreover, WM tasks have not been studied in young Malay adults, although their everyday spoken language might be influenced in yetunknown ways by levels of background noise in the environment. The aim of this study was to identify the areas in the brain that are activated when normal hearing participants are required to listen to babble noise and perform word-based BRTs in quiet and in 5-dB SNR. Our key hypothesis was that if 5-dB SNR is within the range of enhancement effects, we should see (1) better performance in the WM task in noise as compared to in quiet, and (2) evidence of differential subcortical activation on these two conditions, taking into account that with 5-dB SNR, the hippocampus and cerebellum might increase attention, thereby enhancing the speech signals via SR mechanisms as suggested by previous researchers (Moss et al., 2004; Rousseau \& ChapeauBlondeau, 2004). If our level of noise is beyond that which produces enhancement effects, we should see (1) interference on the WM task in noise as compared to the same task in quiet, and (2) we would infer that a cerebellar/hippocampal-based resonance mechanism is not at play, but rather interference effects might produce increased demands on prefrontal cortical processes.

\section{Materials and methods}

\section{Participants}

Fifteen right-handed (Oldfield, 1971) Malay male participants with ages ranging from 20 to 29 years (mean age: 27 years, SD 1.60) were recruited to participate. All were native Malay speakers and reported no history of psychiatric or neurological disorders and no current use of any psychoactive medications. From self-report assessment, all participants had normal hearing and no history of long-time exposure to loud noises. After full explanation of the nature and risks of the study, informed consent was obtained according to the protocols approved by the Institutional Ethics Committee (IEC) of Universiti Kebangsaan Malaysia. (Reference no: UKM 1.5.3.5/244/ NN-075-2009).

\section{Data acquisition}

Functional MRI scans were conducted in the Department of Radiology, UKM Medical Centre using a 1.5 tesla magnetic resonance imaging (MRI) system (Siemens Avanto) equipped with functional imaging options and echo planar imaging capabilities. A radiofrequency (RF) head coil was used for signal transmission and reception. Prior to each functional imaging scan, a MRI structural scan was obtained using $\mathrm{T} 1 *$-weighted multiplanar reconstruction (MPR) spin-echo pulse sequence with the following parameters: $\mathrm{TR}=1240 \mathrm{~ms}, \mathrm{FOV}=250 \mathrm{~mm}$ $\times 250 \mathrm{~mm}$, flip angle $=90^{\circ}$, matrix size $=128 \times 128$, and slice thickness $=1 \mathrm{~mm}$. Functional images were then acquired using a gradient echo-echo planar imaging (GRE-EPI) pulse sequence. Each whole brain acquisition consisted of 21 axial slices covering the whole brain volume including cerebellum. The following parameters were used during the study: acquisition time $(T R)=2 \mathrm{~s}$, echo time $(\mathrm{TE})=50 \mathrm{~ms}$, field of view $(\mathrm{FOV})=192 \times 192$ $\mathrm{mm}$, flip angle $(\alpha)=90^{\circ}$, matrix size $=128 \times 128$ and slice thickness $=5 \mathrm{~mm}$ with $1.25 \mathrm{~mm}$ gap. A sparse imaging paradigm was used to avoid the interference of scanner sound with the stimulus (Hall et al., 1999).

\section{Development of stimulus and babble noise}

The stimuli consisted of a series of natural speech words produced by a Malay male adult voice and were digitally recorded (Sony digital voice editor), stored and edited (Adobe Audition 2.0) and played with an intensity level of $55 \mathrm{~dB}$. The babble noise stimulus was originally recorded from five volunteers reading difference passages simultaneously and edited so that the intensity level was $50 \mathrm{~dB}$. For the working memory task in noise (WMN), the speech stimuli were embedded within the babble noise. Thus, the signal-to-noise ratio (SNR) of speech information was $5 \mathrm{~dB}$ throughout the presentation.

\section{Experimental paradigm}

Auditory stimuli were presented binaurally during the study. There were 120 trials in total with trials of 16-s duration. There were four different conditions: (i) 20 
trials of listening to noise (N), (ii) 20 trials of performing the WM task in 5-dB SNR (WMN), (iii) 20 trials of performing the WM task in quiet (WMQ), and (iv) 60 trials of rest with no stimuli, thereafter referred to as quiet (Q). The sequence of conditions was fixed: N-Q-WMNQ-WMQ-Q-N because reaction times are faster when using fixed sequences (Hazeltine, 2002). Total scan time was 32 min per participant.

In order to construct an experimental trial for WMQ and WMN conditions, a total of 40 (2-syllable and 3 -syllable) verbs and nouns that were unrelated familiar Malay words were randomized, producing 40-trial sets. Five consecutive stimuli each with 0.6 -s duration separated by 0.5 -s silent gap comprised a 5 -s stimulus train. During a trial, the stimuli were presented at the $6^{\text {th }}$ second and lasted approximately $5 \mathrm{~s}$. For WMQ and WMN conditions, participants were given $5 \mathrm{~s}$ to repeat backward aloud all the words that were presented. The scans were acquired $5 \mathrm{~s}$ after the stimulus and continuously through the recall (retrieval) phase of the WM task.

\section{fMRI and behavioral procedures}

Before performing the fMRI scans, instructions about the task were explained in detail to the participants. In order to avoid systematic behavioral confounds, all participants were familiarized with the task using a training program outside of the scanner (containing trials that were identical to those used during scanning). During the fMRI scan, participants were instructed to focus on the tasks given and to remain still. During scanning, participants lay comfortably in a supine position with an adjusted head holder restricting head movement. Auditory stimuli were presented through earphones. During scanning, each individual participant's scores were recorded manually by an experimenter in the console room (i.e., number of correct backward repetition trials).

\section{Data analysis}

Paired t-tests were used to analyze behavioral data (in terms of accuracy) on the word-based BRT in quiet and in noise. fMRI data were analyzed using MATLAB 7.4-R2008a (MathWorks Inc., Natick, MA, USA) and Statistical Parametric Mapping (SPM8) (Functional Imaging Laboratory, Wellcome Department of Imaging Neuroscience, Institute of Neurology, University College of London, UK; http://www.fil.ion.ucl.ac.uk/spm). The first two volumes of the functional scans were discarded. The functional scans were pre-processed using movement correction, 12-parameter nonlinear normalization into the MNI-reference state as implemented in SPM8, and smoothing $(\mathrm{FWHM}=8 \mathrm{~mm})$.

The fMRI data were analyzed according to the general linear model as implemented in SPM8. Three regressors were included in the design: (i) WMQ, (ii) WMN, and (iii) $\mathrm{N}$. The regressors were convolved using the hemodynamic response function. The activated brain regions during the retrieval phase in WM tasks were examined using
$\mathrm{WMN}$, (ii) $\mathrm{WMQ}$, (iii) $\mathrm{N}$, (iv) $\mathrm{WMQ}>\mathrm{WMN}$, and (v) $\mathrm{WMN}>\mathrm{WMQ}>\mathrm{N}$. The contrasts were computed for each participant in the first level fixed effects analysis. Contrast images were then entered into the second level group random-effects analysis. A cortical brain region is regarded as significantly activated only if a minimum cluster size of 10 voxels is reached at $P_{\text {FWEcorr }}<0.001$ (cluster level). All voxels or clusters with t-values higher than 3.5 were included in region of interest analysis (ROI) using WFU PickAtlas (Maldjian et al., 2003).

The laterality index (LI) was calculated using the following formula:

$$
\mathrm{LI}=\left(\mathrm{V}_{\mathrm{L}}-\mathrm{V}_{\mathrm{R}}\right) /\left(\mathrm{V}_{\mathrm{L}}+\mathrm{V}_{\mathrm{R}}\right)
$$

where $\mathrm{V}_{\mathrm{L}}$ is the number of the activated voxels in the left hemisphere, and $V_{R}$ is the number of activated voxels in the right hemisphere. LI values could range from -1 to 1 , with -1 to 0 indicating right hemisphere dominance and 0 to 1 indicating left hemisphere dominance (Seghier, 2008).

\section{Results}

\section{Behavioral scores}

Participants scored significantly better (more accurately) during the word-based backward repeat task (word-based BRT) in 5-dB SNR as compared to the same task in quiet (Table 1). This was verified using a paired $t$-test revealing a significant difference between behavioral scores in the word-based BRT in quiet and in noise conditions $(p<0.027, t=-2.475, \mathrm{df}=14)$.

Table 1. Demographic and performance data obtained from 15 participants

\begin{tabular}{ll}
\hline Participants & \\
\hline$n$ & 15 \\
Age (range) & $20-29$ \\
Age (mean $\pm \mathrm{SD})$ & $27.0 \pm 1.6$ \\
Years of education (mean $\pm \mathrm{SD})$ & $14.9 \pm 8.1$ \\
Word-based BRT (WMQ), accuracy rate $(\% \pm \mathrm{SD})$ & $46.3 \pm 30.0$ \\
Word-based BRT $(\mathrm{WMN})$, accuracy rate $(\% \pm \mathrm{SD})$ & $53.7 \pm 4.2$ \\
\hline
\end{tabular}

WMQ, working memory task in quiet; WMN, working memory task in noise.

\section{fMRI results}

\section{Listening to babble noise ( $N$ )}

Table 2 depicts details of the activation characteristics shown in Figure 1(a) including the number of activated voxels (NOV), coordinates of maximum intensity, $p$-values and the respective areas. Significant activation in bilateral superior temporal gyrus (STG) (left $t=32.82$, right $t=22.5$ ) and middle temporal gyrus (MTG) (left $t$ $=27.43$, right $t=12.12$ ) were observed. Result shows rightward asymmetries in both regions. 
Table 2. Anatomical area, brain hemisphere, t-value, coordinates of maximum intensity $(\mathrm{x}, \mathrm{y}, \mathrm{z})$ and number of activated voxels obtained from group analysis $(n=15$, $p<0.05)$ during listening to babble noise (N) minus quiet condition (baseline)

\begin{tabular}{ccccc}
\hline $\begin{array}{c}\text { Anatomical } \\
\text { area }\end{array}$ & Hemisphere & t-value & $\begin{array}{c}\text { Coordinate } \\
(\mathbf{x}, \mathbf{y}, \mathbf{z} \mathbf{m m})\end{array}$ & NOV \\
\hline STG & R & 22.5 & $62,-10,-2$ & 1829 \\
& L & 32.82 & $-58,-20,4$ & 1649 \\
\multirow{2}{*}{ MTG } & R & 12.12 & $68,-34,0$ & 906 \\
& L & 27.43 & $-58,-20,0$ & 861 \\
\hline
\end{tabular}

NOV, number of activated voxels; STG, superior temporal gyrus; MTG, middle temporal gyrus; L, left; R, right.
Working memory task in quiet (WMQ) and working memory task in noise (WMN)

Comparing between WMQ and WMN conditions, all regions of interest (ROIs) commonly associated with the retrieval phase in WM demonstrate higher BOLD activation in the WMQ condition except bilateral STG (WMQ; left $t=25.07$, right $t=22.69$, WMN; left $t=31.11$, right $t=24.48$ ), hippocampus (WMQ; right $t=4.91$, WMN; left $t=5.22$, right $t=$ 5.83), anterior cerebellum (WMQ; left $t=8.42$, right $t$ $=11.82, \mathrm{WMN}$; left $t=9.98$, right $t=12.08)$ and right posterior cerebellum (WMQ; left $t=5.71$, right $t=$ 12.47, WMN; left $t=5.17$, right $t=12.75$ ) as in Table 3, Figure 1(b) and Figure 1(c). The extent of activation

Table 3. Anatomical area, brain hemisphere, t-value, coordinates of maximum intensity $(\mathrm{x}, \mathrm{y}, \mathrm{z})$ and number of activated voxels $(\mathrm{n}=15, p<0.05)$ obtained from Working Memory task in Quiet (WMQ) minus quiet (baseline) condition and the Working Memory task in Noise (WMN) minus quiet (baseline)

\begin{tabular}{|c|c|c|c|c|c|c|c|}
\hline \multicolumn{4}{|c|}{ WMQ minus QUIET } & \multicolumn{4}{|c|}{ WMN minus QUIET } \\
\hline Anatomical area & Hemisphere & t-value & $\begin{array}{l}\text { Coordinate } \\
(\mathrm{x}, \mathrm{y}, \mathrm{z} \mathbf{m m})\end{array}$ & NOV & t-value & $\begin{array}{l}\text { Coordinate } \\
(\mathbf{x}, \mathbf{y}, \mathbf{z} \text { mm })\end{array}$ & NOV \\
\hline \multirow{2}{*}{ STG } & $\mathrm{R}$ & 22.69 & $64,14,-4$ & 2109 & 24.48 & $64,-14,-4$ & 2114 \\
\hline & $\mathrm{L}$ & 25.07 & $-60,-20,2$ & 1839 & 31.11 & $-58,-20,2$ & 1871 \\
\hline \multirow{2}{*}{ PCG } & $\mathrm{R}$ & 15.1 & $50,-6,34$ & 1006 & 15.9 & $50,-6,34$ & 971 \\
\hline & $\mathrm{L}$ & 14.62 & $-54,-8,30$ & 1311 & 14.86 & $-52,-8,30$ & 1280 \\
\hline \multirow{2}{*}{ MTG } & $\mathrm{R}$ & 16.61 & $64,16,-8$ & 1103 & 17.09 & $64,-16,-8$ & 958 \\
\hline & $\mathrm{L}$ & 24.31 & $-60,-18,0$ & 1294 & 29.37 & $-58,-20,0$ & 1208 \\
\hline \multirow{2}{*}{ Lingual gyrus } & $\mathrm{R}$ & 7.51 & $8,-90,-4$ & 115 & 8.35 & $10,-82,-14$ & 115 \\
\hline & $\mathrm{L}$ & 7.33 & $-8,-86,-16$ & 254 & 8.94 & $-8,-86,-16$ & 181 \\
\hline \multirow{2}{*}{ SPL } & $\mathrm{R}$ & 7.44 & $34,-64,54$ & 235 & 6.62 & $34,-64,54$ & 130 \\
\hline & $\mathrm{L}$ & 9.74 & $-30,-66,52$ & 445 & 9.46 & $-30,-64,50$ & 410 \\
\hline \multirow{2}{*}{ IFG (tri) } & $\mathrm{R}$ & 6.05 & $42,18,4$ & 141 & 4.84 & $42,18,4$ & 8 \\
\hline & $\mathrm{L}$ & 11.24 & $-52,16,-2$ & 484 & 12.96 & $-52,16,-2$ & 206 \\
\hline \multirow{2}{*}{ MFG } & $\mathrm{R}$ & 9.12 & $38,42,24$ & 623 & 8.9 & $38,42,24$ & 365 \\
\hline & $\mathrm{L}$ & 8.52 & $-46,10,36$ & 583 & 8.55 & $-34,52,20$ & 523 \\
\hline \multirow{2}{*}{ Precuneus } & $\mathrm{R}$ & 7.65 & $6,-70,48$ & 208 & 6.76 & $6,-68,48$ & 116 \\
\hline & $\mathrm{L}$ & 7.55 & $-6,-70,48$ & 346 & 7.07 & $-6,-46,72$ & 279 \\
\hline \multirow{2}{*}{ Post-CG } & $\mathrm{R}$ & 17.02 & $52,-8,30$ & 729 & 17.91 & $52,-8,30$ & 669 \\
\hline & $\mathrm{L}$ & 16.93 & $-56,-8,24$ & 1279 & 15.99 & $-54,-10,26$ & 1166 \\
\hline \multirow{2}{*}{ Hippocampus } & $\mathrm{R}$ & 4.91 & $16,-28,-8$ & 6 & 5.83 & $16,-28,-8$ & 11 \\
\hline & $\mathrm{L}$ & - & - & - & 5.22 & $-16,-34,10$ & 33 \\
\hline \multirow{2}{*}{ Anterior cerebellum } & $\mathrm{R}$ & 11.82 & $26,-58,-28$ & 314 & 12.08 & $22,-60,-26$ & 327 \\
\hline & $\mathrm{L}$ & 8.42 & $-30,-58,-30$ & 231 & 9.98 & $-32,-58,-32$ & 354 \\
\hline \multirow{2}{*}{ Posterior cerebellum } & $\mathrm{R}$ & 12.47 & $26,-62,-28$ & 1714 & 12.75 & $24,-62,-28$ & 2088 \\
\hline & $\mathrm{L}$ & 5.71 & $-28,-38,-46$ & 12 & 5.17 & $-28,-38,-46$ & 11 \\
\hline
\end{tabular}

WMQ, working memory task in quiet; WMN, working memory task in noise; NOV, number of activated voxels; STG, superior temporal gyrus; MTG, middle temporal gyrus; PCG, precentral gyrus; SPL, superior parietal lobes; IPL, inferior parietal lobes; IFG (tri), inferior frontal gyrus (triangularis); MFG, middle frontal gyrus; Post-CG, postcentral gyrus; L, left; R, right. 

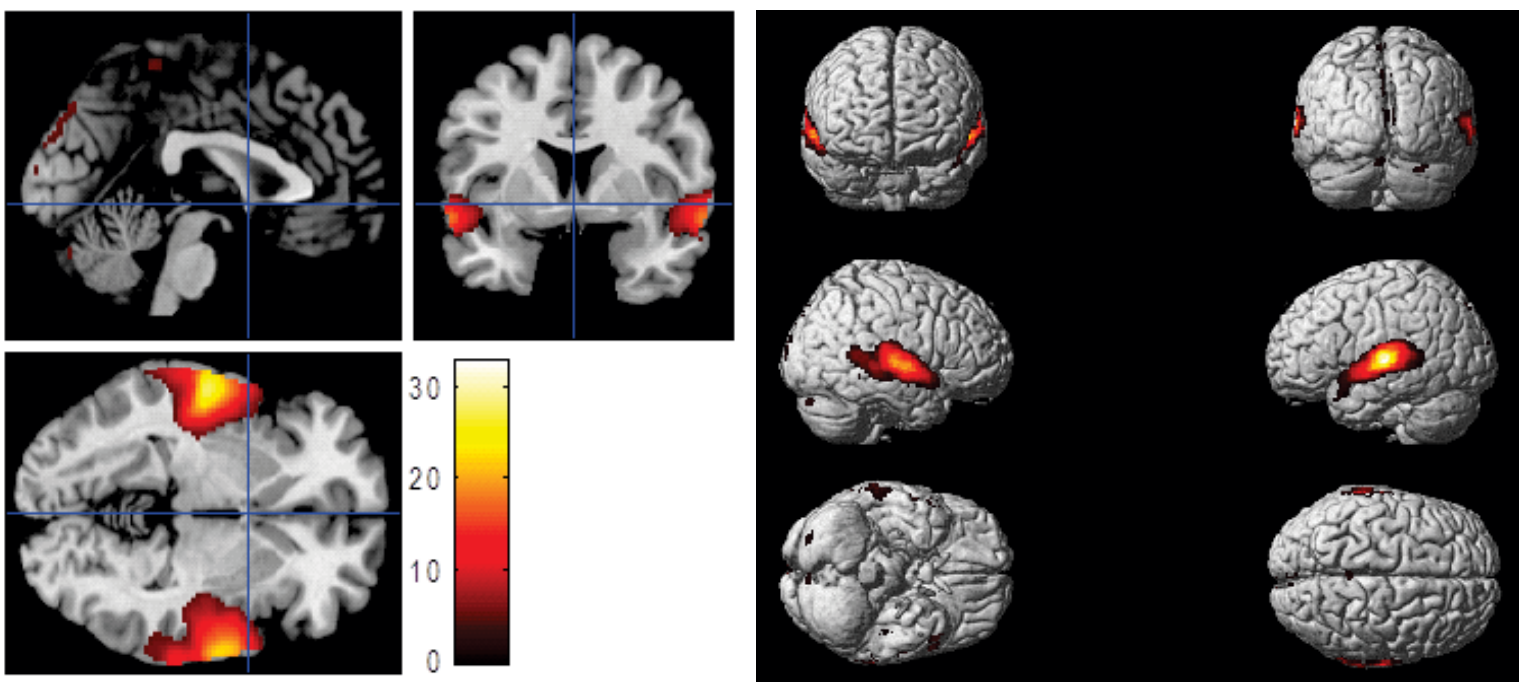

(a) Noise > Quiet
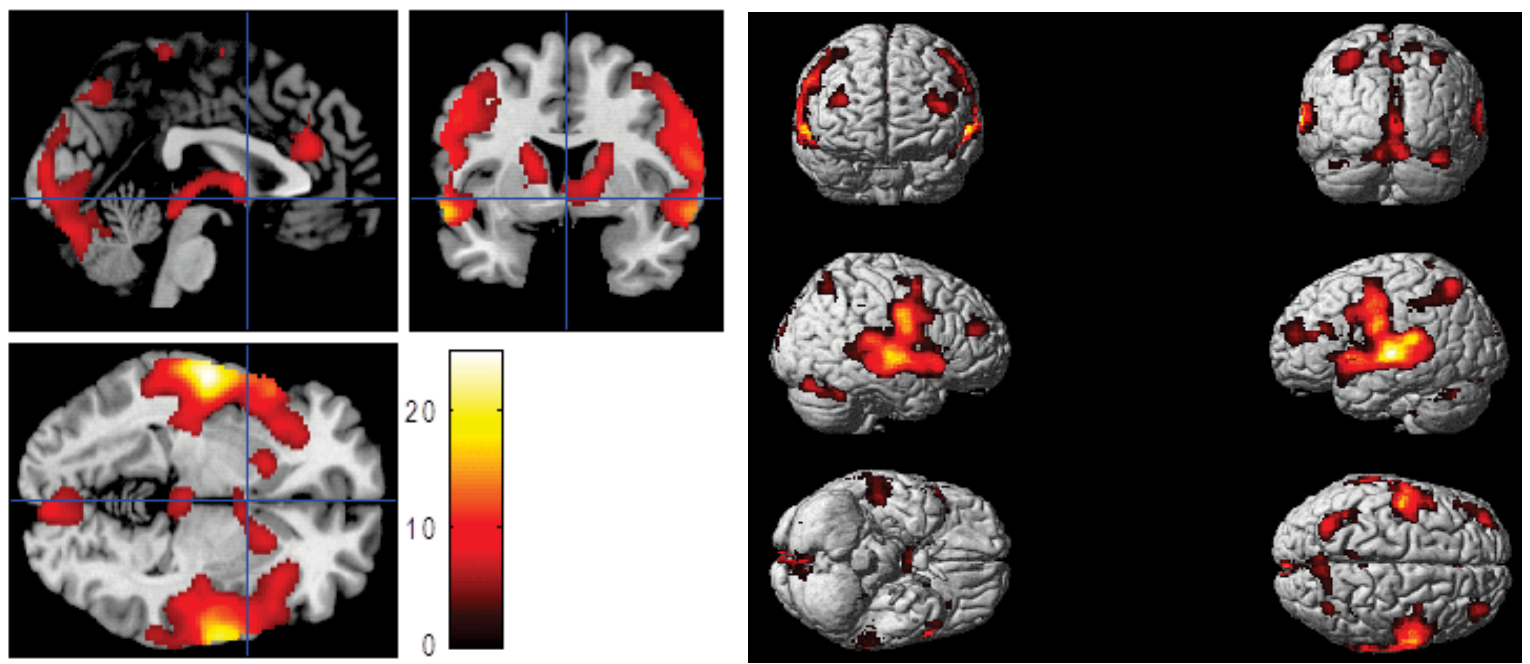

(b) Working Memory Task in Quiet (WMQ) > Quiet
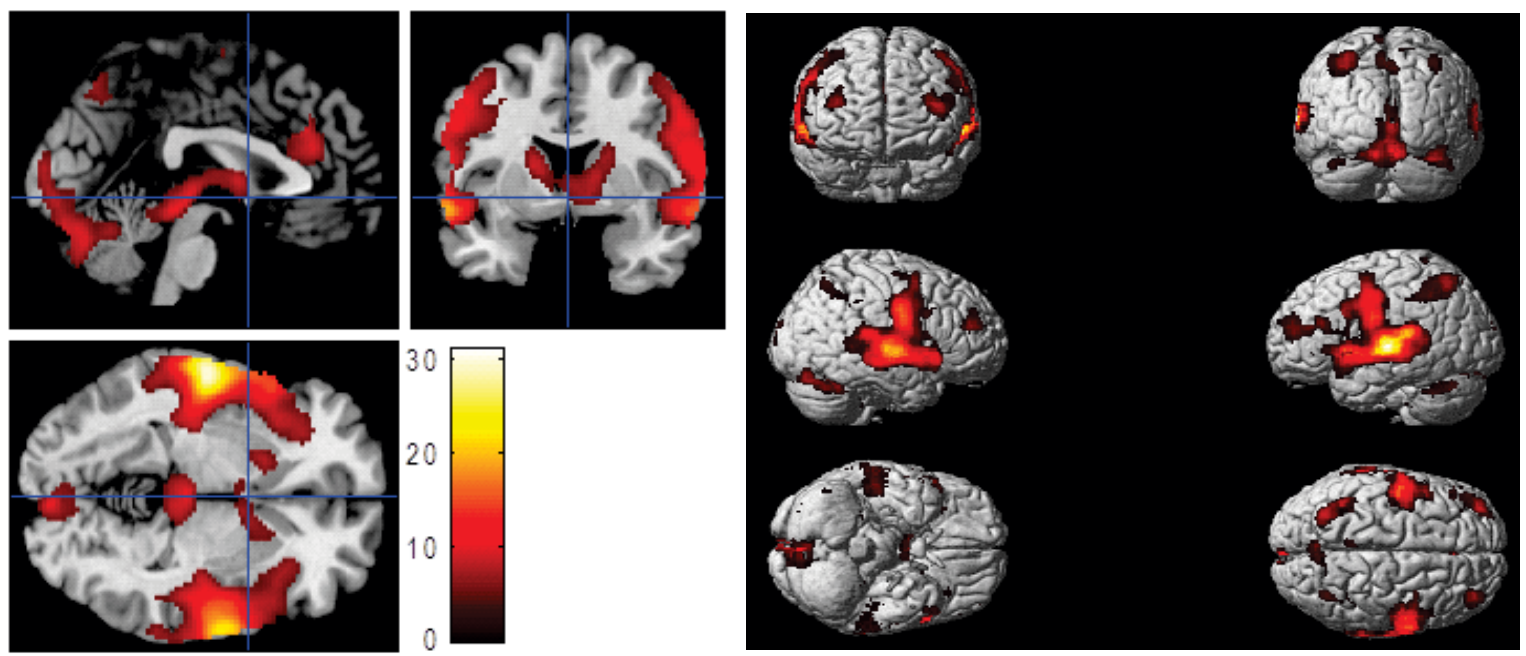

(c) Working Memory Task in Noise (WMN) > Quiet

Figure 1. Statistical parametric maps (SPMs) obtain from group analysis $(n=15, p<0.05)$ showing brain activation associated with (a) N > Q, (b) WMQ > Q and (c) WMN $>$ Q, overlaid onto structural brain images, shown for transverse, sagittal, and coronal slices (left side of figures), and using a whole brain map (right side of figures). The t-values for the activated voxels are scaled to the colors as shown. [Note: left side of the brain is on the left: neurological conventions]. 
in these regions during $\mathrm{WMN}$ is further supported by the contrast WMN $>$ WMQ (Table 4, Figure 2a and Figure $2 \mathrm{~b}$ and the double subtraction analysis, WMN $>$ WMQ $>$ N (Table 5 and Figure 3). Notably, previous studies using digit BRT do not report such activation (Zhou et al., 2006; Sun et al., 2005).

\section{Behavioral scores in relation to fMRI results}

Correlations between behavioral scores on the WMQ and WMN tasks (separately) and fMRI activation in eight ROIs were examined for both right and left sides of STG, PCG, hippocampus, and cerebellum to assess a possible relationship between behavior and brain activity. Correlations ranged from -0.174 to 0.395 for the WMQ condition and between -0.232 and 0.325

Table 4. Anatomical area, brain hemisphere, $t$-value, coordinates of maximum intensity $(\mathrm{x}, \mathrm{y}, \mathrm{z})$ and number of activated voxels are obtained from the group analysis $(n=15, p<0.001)$ comparing the Working Memory task in Noise (WMN) minus Working Memory task in Quiet (WMQ) as shown in Figure 3

\begin{tabular}{ccccc}
\hline \multicolumn{5}{c}{ WMN > WMQ } \\
\hline $\begin{array}{c}\text { Anatomical } \\
\text { area }\end{array}$ & Hemisphere & t-value & $\begin{array}{c}\text { Coordinate } \\
(\mathbf{x}, \mathbf{y}, \mathbf{z} \text { mm })\end{array}$ & NOV \\
\hline STG & $\mathrm{L}$ & 7.01 & $-54,-22,4$ & 562 \\
$\begin{array}{c}\text { Cerebellar } \\
\text { vermis }\end{array}$ & $\mathrm{R}$ & 4.53 & $0,-44,-8$ & 95 \\
STG & $\mathrm{R}$ & 4.44 & $50,-20,2$ & 131 \\
Cerebellum & $\mathrm{R}$ & 3.9 & $2,-86,-28$ & 22 \\
Cerebellum & $\mathrm{L}$ & 3.83 & $-8,-40,-24$ & 22 \\
Cerebellum & $\mathrm{R}$ & 3.79 & $10,-38,-22$ & 30 \\
Hippocampus & $\mathrm{L}$ & 3.62 & $-12,-36,10$ & 43 \\
\hline
\end{tabular}

WMQ, working memory task in quiet; WMN, working memory task in noise NOV, number of activated voxels; STG, superior temporal gyrus; L, left; R, right.
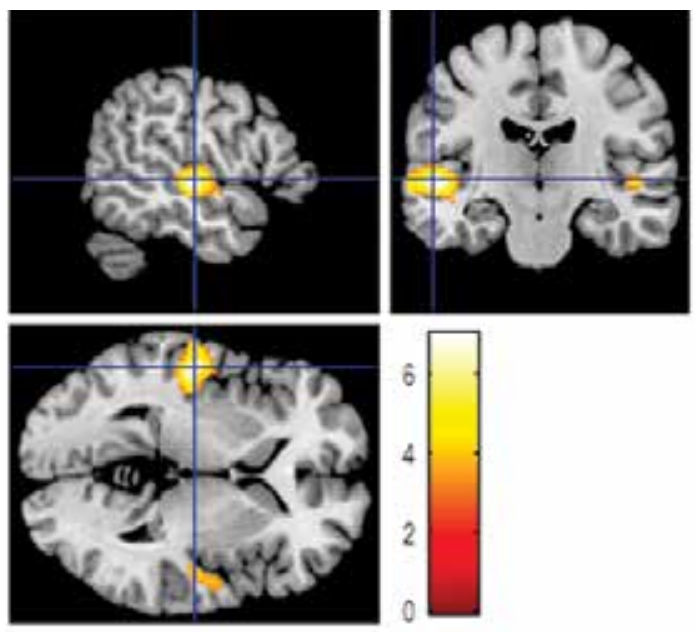

(a) WMN minus WMQ conditions: transverse, sagittal and coronal slices for the WMN condition, but none were statistically significant $(p>0.05)$. In the WMQ condition the correlation shown for the left STG was the highest (0.395) and for the WMN condition the correlation shown for the left cerebellum was the highest (0.325), but even those were not statistically significant.

\section{Discussion}

The main purpose of this study was to examine the areas of the brain that are activated in the three listening conditions while focusing on our specific aims of examining whether the 5-dB SNR is within the range to cause enhancement effects through a stochastic resonance (SR) mechanism or beyond the range to produce interference.

Table 5. Anatomical area, brain hemisphere, $t$-value, coordinates of maximum intensity $(\mathrm{x}, \mathrm{y}, \mathrm{z})$ and number of activated voxels are obtained from the group analysis $(n=15, p<0.001)$ on double subtraction; comparing the Working Memory task in Noise (WMN) minus Working Memory task in Quiet (WMQ) minus Noise $(\mathrm{N})$ conditions as shown in Figure 3

\begin{tabular}{ccccc}
\hline \multicolumn{5}{c}{ WMN minus WMQ minus N } \\
\hline $\begin{array}{c}\text { Anatomical } \\
\text { area }\end{array}$ & Hemisphere & t-value & $\begin{array}{c}\text { Coordinate } \\
\mathbf{( x ,}, \mathbf{y}, \mathbf{z} \text { mm) }\end{array}$ & NOV \\
\hline $\begin{array}{c}\text { Hippocampus } \\
\text { SFG }\end{array}$ & $\mathrm{R}$ & 7.69 & $24,-8,-30$ & 30 \\
SFG & $\mathrm{R}$ & 7.63 & $16,-12,40$ & 44 \\
Cerebellum & $\mathrm{R}$ & 5.96 & $2,30,48$ & 48 \\
Cerebellum & $\mathrm{L}$ & 5.44 & $20,2,66$ & 11 \\
Cerebellum & $\mathrm{L}$ & 4.64 & $-10,-52,-10$ & 37 \\
Cerebellum & $\mathrm{R}$ & 4.49 & $8,-46,-10$ & 34 \\
\hline
\end{tabular}

WMQ, working memory task in quiet; WMN, working memory task in noise; NOV, number of activated voxels; SFG, superior frontal gyrus; L, left; R, right.

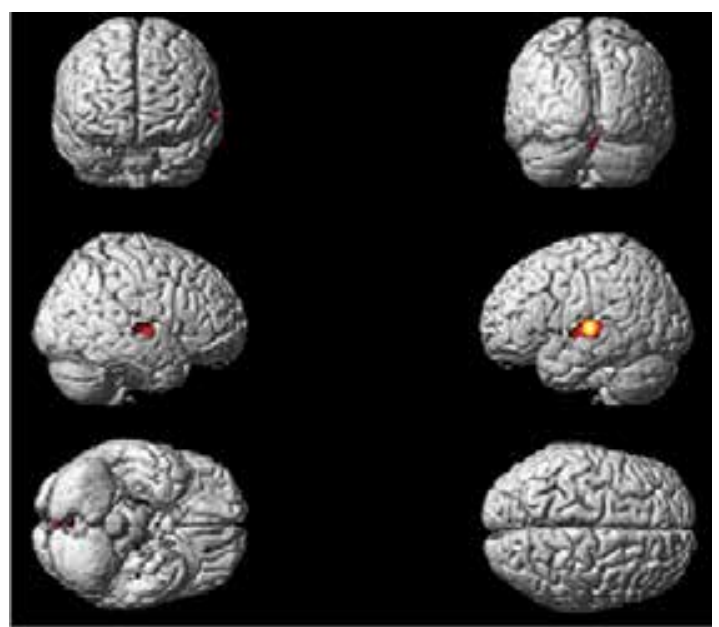

(b) WMN minus WMQ conditions: in whole brain.

Figure 2. Statistical parametric maps (SPMs) obtained from the group analysis $(n=15, p<0.001)$ showing WMN $>$ WMQ. Arrow shows the higher maxima intensity; superior temporal gyrus $(-54,-22,4)$. The t-values for the activated voxels are scaled in colours (red to white) as shown. [Note: left side of the brain is on the left, neurological conventions]. 


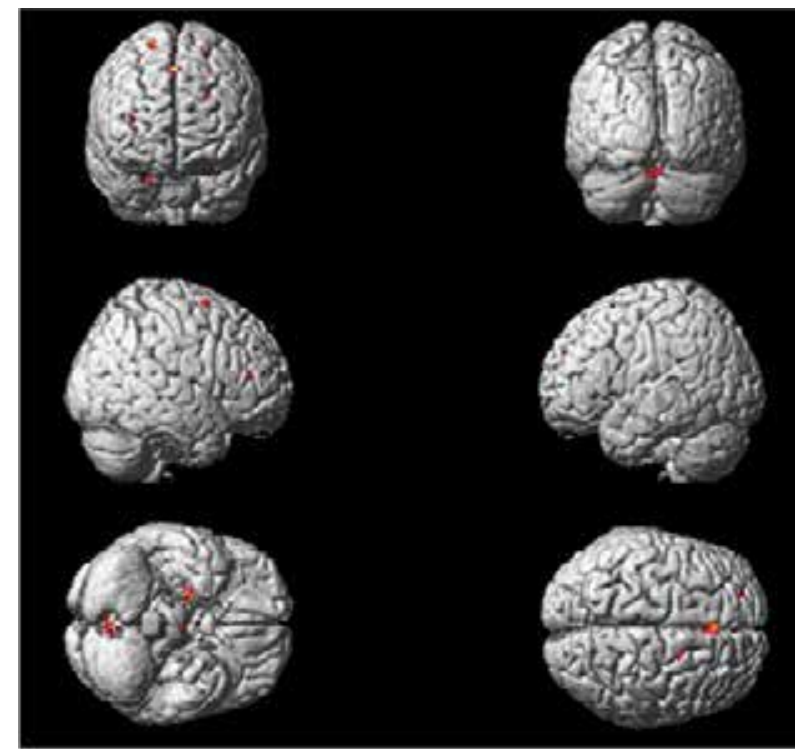

Figure 3. Statistical parametric maps (SPMs) obtained from the group analysis $(n=15, p<0.001)$ showing double subtraction analysis; WMN $>W M Q>N$. [Note: left side of the brain is on the left, neurological conventions].

\section{fMRI}

\section{Listening to babble noise (N)}

Compared to WMQ and WMN, N condition imposed the smallest processing demands as the participants needed only to listen to the babble noise presented binaurally. Activation of bilateral STG and MTG were expected given that auditory stimuli were used, and these regions are primarily involved in auditory processing (Burton \& Small, 2006). Both areas showed rightward asymmetries, supporting the finding that the right hemisphere is relatively more sensitive than the left in the processing of nonverbal stimuli (Sequeira et al., 2008).

\section{Comparing WMQ and WMN}

Result reveals the following primary effects: 1) cortical areas with significant activation during WMQ are similar to those demonstrated in WMN. This suggests that WMQ and WMN use the same neural areas in processing both tasks. 2) In WMN, results show decreases in neural activity in all ROIs except in the cerebellum and hippocampus, which points to interesting findings in relation to the use 5-dB SNR in word stimuli (which will be discussed later).

In both the WMQ and WMN conditions (as shown in Table 3 and Figure $1 \mathrm{~b}$ and 1c), there was bilateral STG and MTG activation, consistent with previous studies assessing the involvement of these areas in auditory (Burton \& Small, 2006; Jonides et al., 1998) and spoken word processing (Wong et al., 2009). Both tasks also activated the prefrontal cortex, specifically the MFG and IFG covering BA 47 bilaterally. These areas are suggested to play a crucial role in executive functioning during WM tasks (Jonides et al., 1998). PCG and post-CG were also activated, and these areas are important for rehearsal of verbal information (Smith et al., 2000). As we asked participants to remember words, the storage area as suggested by Shivde \& ThompsonSchill (2004) was activated (SPL, IPL and precuneus) (Hodge et al., 2000). SPL and IPL covering BA 40 and BA 7 are also proposed to be involved in encoding and retrieval of verbal information (Luck et al., 2010; Sweet et al., 2008; Jonides et al., 1998). Activation in the lingual gyrus was also found, consistent with the proposal that the area is involved in information encoding (Karlsgodt et al., 2005). These tasks also activated the hippocampus and cerebellum. Traditionally, the hippocampus and cerebellum have been thought to be responsible for motor coordination and balance but recently these structures are hypothesized to be involved in executive function and attention during cognitive processing (Gottwald et al., 2003; Desmond \& Fiez, 1998). We postulate that both WMQ and WMN activated the same neural areas because both tasks involved the requirements to attend to stimuli and repeat them backwards. Both tasks involved storage, rehearsal, encoding, and executive functioning (Baddeley \& Wilson, 2002; Baddeley, 1986; Baddeley \& Hitch, 1974), all of which depend on attention.

The extended activation in the bilateral anterior cerebellum and right posterior cerebellum during WMN suggests greater recruitment of attention resources, probably to compensate for interference due to $5-\mathrm{dB}$ SNR. This finding supports the proposal based on previous work in other domains that, in the presence of relatively low-level background noise, participants' attention to word stimuli is enhanced via SR mechanisms (Moss et al., 2004) involving a hippocampal-cerebellar system. A related possibility is that the presence of 5-dB SNR might prime the system to work better, a hypothesis supported by earlier claims that humans are thought to have the special ability to focus and pay more attention to particular sounds given in a mixture of various sounds (Sohn \& Lee, 2000). Another possibility is that the cerebellum amplifies and refines the signal to facilitate correct decision making when the level of background noise is suitable within the range of the enhancement phenomenon. These possibilities, which are not mutually exclusive, are further supported by our behavioral scores indicating that participants responded better in the presence of 5-dB SNR (Table 1). The proposed involvement of the cerebellum in attention is also supported by previous researchers in other domains (Schweizer et al., 2007; Krischen et al., 2006; TederSalejarvi et al., 2005; Gottwald et al., 2003; Townsend et al., 2001; Harris et al., 1999; Courchesne, 1997).

The hippocampus was also activated in the present study although the NOV (Table 3, Figure 2 and Figure 3 ) is small, and the involvement of the hippocampus in attention is implicated in an earlier study (Faraco et al., 2011; Toepper et al., 2010). We suggest that activation of the hippocampus in the present study is not due to the WM mechanism but rather to the role of the hippocampus as an attention controller in compensating for the effects of 
noise. In this manner, the hippocampus and cerebellum might work together in the presence of background noise to increase attention to relevant stimuli (i.e., the signal) via SR mechanisms. This hypothesis is supported by our fMRI results in that the left hippocampus was activated only in WMN conditions and not in the WMQ condition. Furthermore, the nature of stimuli used in the present study was not similar to that of previous studies (Lindauer et al., 2005; Karlsgodt et al., 2005) that have investigated the WM system, reporting activation of the hippocampus. Additionally, behavioral results in the present study revealed that participants performed better with noise (i.e., in WMN conditions). Thus, our study clearly differs from previous studies both in the task and in the findings, pointing to hippocampal involvement in SR mechanisms involved in attention rather than to the WM processes implicated in those previous studies. The involvement of hippocampus as an attention controller is further supported by patients with posttraumatic stress disorder (PTSD) (Lindauer et al., 2006) and ADHD (Plessen et al., 2006).

In summary, comparing the WMQ and WMN tasks of the present study, results demonstrate higher BOLD activity in the WMQ condition except in the bilateral anterior cerebellum, right posterior cerebellum, and left hippocampus. Findings of other activated areas in this study such as PCG, MTG, lingual gyrus, SPL, IFG (triangular), MFG, precuneus, and post-CG (Table 3) appear at odds with previous studies suggesting that performing a WM task in noise increases neural activity related to WM demands (Just et al., 1996; Wong et al., 2004). We propose that the differences between the present and previous studies (Just et al., 1996; Wong et al., 2004) reveal the operation of a SR mechanism in the present study. The idea of SR is relatively new in neurological science, suggesting that, in the presence of external noise up to a finite level of intensity, there is an enhanced sensitivity to the stimuli (the signal) (Moss et al., 2004). We postulate that the 5 -dB SNR used in the present study was within the range to produce such enhancement effects as shown in our task. These findings also support the idea that brain functions require additional attention resources under noisy conditions. Our results are further supported by the behavioral scores demonstrating that participants performed better in the presence of noise than without it (quiet). Whether or not our participants would be hindered in performance with higher levels of noise remains to be studied, but we can safely conclude on the basis of the present findings that they show enhancement effects with such relatively low levels of noise, thereby providing a firm foundation for future studies of this type.

The manipulation of noise in the present study is very important given that noise pollution is now one of the fast-becoming major public health issues in Malaysia (Ismail et al., 2009). Yusoff \& Ishak (2005) discovered that noise level exposure experienced by the Klang Valley residents exceeded the Malaysian Department of
Environment's (DOE) guidelines on a daily basis. In the next 5 to 10 years, noise levels are expected to increase and this issue will become a major concern. However, the cerebellar-hippocampus involvement as proposed in this study has not yet been conclusive and evidence is still limited especially in regard to attention and WM. Thus, future study related to this issue is needed.

\section{Conclusions}

There are a number of similarities in task and brain activation in the present study compared to other available studies. However, the present study highlights the involvement of the cerebellum and hippocampus in a SR mechanism when a BRT is performed in the presence of a low level (5-dB SNR) of background noise. The present study provides evidence that (1) the 5-dB SNR used in our study is within the range of enhancement phenomena best explained with SR mechanisms. This is supported by the behavioral scores revealing better performance in the WMN condition and is further supported by our fMRI results revealing activation in the bilateral posterior cerebellum, left anterior cerebellum, and left hippocampus, all of which are relevant in attention processes and have been implicated in SR mechanisms. However, while we speculate about possible underlying effects on SR mechanisms in association with cerebellarhippocampal activation, it is clear that additional research is necessary to cement such claims (or refute them). The present study, therefore, provides a foundation and adds to the very limited literature on the role of the cerebellum and hippocampus in attention enhancement effects in the context of cognitive performance.

\section{Acknowledgments}

We thank Sa'don Samian from the Department of Radiology, Universiti Kebangsaan Malaysia Medical Centre for the assistance in fMRI scans; Noorazrul Azmie Yahya, Aini Ismafairus Abd. Hamid, Khairiah Abdul Hamid, and Mazlyfarina Mohamad from Diagnostic Imaging and Radiotherapy Department, Universiti Kebangsaan Malaysia for providing insight and ideas. This work is supported by the Research University Grant UKM GUP-SK-07-020-205.

\section{References}

Alexander, M. P., Gillingham, S., Schweizer, T., \& Stuss, D. T. (2011). Cognitive impairments due to focal cerebellar injuries in adults. Cortex, in press (corrected proof). Available online 14 April 2011.

Baddeley, A. D., \& Hitch, G. J. (1974). The psychology of learning and motivation, vol 8 (pp. 47-89). New York: Academic Press.

Baddeley, A. D. (1986). Working memory. New York: Oxford University Press.

Baddeley, A. D. (1992). Working memory. Science 9, 255-556.

Baddeley, A. D. (2000). The episodic buffer: A new component of working memory. Trends in Cognitive Science 4, 23-417.

Baddeley, A. D., \& Wilson, B. A. (2002). Prose recall and amnesia: Implications for the structure of working memory. Neuropsychologia 40, 1737-1743.

Baddeley, A. D. (2003a). Working memory: Looking back and looking forward. Nature Reviews Neuroscience 10, 829-839.

Baddeley, A. D. (2003b). Working memory and language: An overview. Journal of Communication Disorders 36, 189-208. 
Baddeley, A. D., Allen, R., \& Vargha-Khadem, F. (2010). Is the hippocampus necessary for visual and verbal binding in working memory? Neuropsychologia 48, 1089-1095.

Biederman, J., \& Spencer, T. (1998). Attention-deficit/hyperactivity disorder (ADHD) as a noradrenergic disorder. Biological Psychiatry 46, 1234-1242.

Bledsoe, J., Semrud-Clikeman, M., \& Pliszka, S. (2009). A magnetic resonance imaging study of the cerebellar vermis in chronically treated and treatment-naïve children with attention deficit/ hyperactivity disorder combined type. Biological Psychiatry 65, 620-624.

Booth, J. R., Wood, L., Lu, D., Houk, J. C., \& Bitan, T. (2007). The role of the basal ganglia and cerebellum in language processing. Brain Research 1133, 136-144.

Brattico, E., Kujala, T., Tervaniemi, M., Alki, P., Ambrosi, L., \& Monitillo, V. (2005). Long-term exposure to occupational noise alters the cortical organization of sound processing. Clinical Neurophysiology 116, 190-203.

Burton, M. W., \& Small, S. L. (2006). Functional neuroanatomy of segmenting speech and nonspeech. Cortex 42, 644-651.

Courchesne, E. (1997). Brainstem, cerebellar and limbic neuroanatomical abnormalities in autism. Current Opinion in Neurobiology 7, 269-278.

Courtney, S. M., Petit, L., Haxby, J. V., \& Ungerleider, L. G. (1998). The role of prefrontal cortex in working memory: Examining the contents of consciousness. Philosophical Transactions of the Royal Society of London. Series B, Biological Sciences 353, 1819-1828.

Desmond, J. E., \& Fiez, J. A. (1998). Neuroimaging studies of the cerebellum: Language, learning and memory. Trends in Cognitive Science 2, 355-362.

Dos Santos Sequeira, S., Specht, K., Hamalainen, H., \& Hugdahl, K. (2008). The effects of background noise on dichotic listening to consonant-vowel syllables. Brain Language 107, 11-15.

Faraco, C. C., Unsworth, N., Langley, J., Terry, D., Li, K., Zhang, D., Liu, T., \& Miller, L. S. (2011). Complex span tasks and hippocampal recruitment during working memory. NeuroImage 55, 773-787.

Fliessbach, K., Trautner, P., Quesada, C. M., Elger, C. E., \& Weber, B. (2007). Cerebellar contributions to episodic memory encoding as revealed by fMRI. Neurolmage 35, 1330-1337.

Gerton, B. K., Brown, T. T., Meyer-Lindenberg, A., Kohn, P., Holt, J. L., Olsen, R. K., Berman, K. B. (2004). Shared and distinct neurophysiological components of the digits forward and backward tasks as revealed by functional neuroimaging. Neuropsychologia 42, 1781-1787.

Gottwald, B., Mihajlovic, Z., Wilde, B., \& Mehdorn, H. M. (2003). Does the cerebellum contribute to specific aspects of attention. Neuropsychologia 41, 1452 -1460.

Hall, A. D., Haggard, P. M., Akeroyd Summerfield, P. R., Elliott, Q., Gurney, M., \& Bowtell, R. W. (1999). "Sparse" temporal sampling in auditory fMRI. Human Brain Mapping 7, 213-223.

Hazeltine, E. (2002). The representation nature of sequence learning: Evidence for goal-based codes. Oxford: Oxford University Press.

Harris, N. S., Courchesne, E., Townsend, J., \& Carper, R. A. (1999). Neuroanatomic contributions to slowed orienting of attention in children in autism. Cognitive Brain Research 8, 61-71.

Henry, L. A., \& Millar, S. (1991). Memory span increase with age: A test of two hypotheses. Journal of Experimental Child Psychology $51,459-484$.

Hodge, S. M., Makris, N., Kennedy, D., Howard, J., McGrath, L., Steele, S., ... Harris, G. J. (2010). Cerebellum, language, and cognition in autism and specific language impairment. Journal of Autism and Developmental Disorders 40, 300-316.

Ismail, A. R., Nor, M. J. M., Mansor, M. R. A., Tahir, M. F. M., \& Zulkifli, R. (2009). Environmental noise assessment and modelling in Malaysia: A comparative monitoring study. European Journal of Scientific Research 20, 236-244.

Jonides, J., Schumacher, E. H., Smith, E. E., Koeppe, R. A., Awh, E., Reuter-Lorenz, P. A., Marshuetz, C., Willis, C. R. (1998). The role of parietal cortex in verbal working memory. Journal of Neuroscience 18, 5026-5034.

Just, M. A., Carpenter, P. A., Keller, T. A., Eddy, W. F., Thulborn K. R. (1996). Brain activation modulated by sentence comprehension. Science 274, 114-116.

Karlsgodt, K. H., Shirinyan, D., Theo, G. M., Cohen, M. S., Connon, T. D. (2005). Hippocampal activation during encoding and retrieval in a verbal working memory paradigm. NeuroImage 25, 1224-1231

Krischen, M. P., Annabel Chen, S. H., Schraedley-Desmond, P., Desmond J. E. (2006). Load- and practice-dependent increases in cerebro-cerebellar activation in verbal working memory: An fMRI Study. NeuroImage 24, 462-472.

Kozou, H., Kujala, B., Shtyrov, Y., Toppila, E., Starck, J., Alku, P., \& Naatanen, R. (2005). The effects of different noise types on speech and non-speech elicited mismatch negativity. Hearing Research 199, 31-39.

Kujala, T., Shtyrov, Y., Winkler, I., Saher, M., Tervaniemi, M., Sallinen, M., ... Näätänen R. (2004). Long-term exposure to noise impairs cortical sound processing and attention control. Psychophysiology $41,875-881$

Kujala, T., \& Brattico, E. (2009). Detrimental noise effects on brain's speech functions. Biological Psychology 81, 135-143.

Light, L. L., \& Anderson, P. A. (1985). Working memory capacity, age and discourse. Journal of Gerontology 40(6), 737-747.

Lindauer, R. J. L., Olff, M., van Meijel, E. P. M., Carlier, I. V. E., \& Gersons, B. P. R. (2005). Cortisol, learning, memory, and attention in relation to smaller hippocampus volume in police officers with posttraumatic stress disorder. Biological Psychiatry 59, 171-177.

Luck, D., Danion, J. M., Marrer, C., Pham, B. T., Gounot, D., \& Foucher, J. (2010). The right parahippocampal gyrus contributes to the formation and maintenance of bound information in working memory. Brain Cognition 72, 255-263.

Maldjian, J. A., Laurienti, P. J., Kraft, R. A., \& Burdette, J. H. (2003). An automated method for neuroanatomic and cytoarchitectonic atlasbased interrogation of fMRI data sets. Neuroimage 19, 1233-1239.

Marvel, C. L. \& Desmond, J. E. (2010). The contributions of cerebrocerebellar circuitry to executive verbal working memory. Cortex 46, 880-895.

Mitchell, K. J., Johnson, M.K., Raye, C.L., \& D’Esposito, M. (2000). fMRI Evidence of age-related hippocampal dysfunction in feature binding in working memory. Cognitive Brain Research 10, 197-206.

Morra, S. (1994). Issues in working memory measurement: testing for M capacity. International Journal of Behavioural Development $17,143$.

Moss, D., Ward, L. M., \& Sannita, W. G. (2004). Stochastic resonance and sensory information processing: A tutorial and review of application. Clinical Neurophysiology 115, 267-281.

Oldfield, R. C. (1971). The assessment and analysis of handedness: The Edinburgh Inventory. Neuropsychologia 9, 97-113.

Plessen, K. J., Bansal, R., Zhu, H., Whiteman, R., Amat, J., Quackenbush, G. A., ... Peterson, S. (2006). Hippocampus and amygdala morphology in attention-deficit/hyperactivity disorder. Archives of General Psychiatry 63, 795-807.

Rousseau, D., \& Chapeau-Blondeau, F. (2004). Suprathreshold stochastic resonance and signal-to-noise ratio improvement in arrays of comparators. Physics Letters 321, 280-290.

Schweizer, T. A., Alexander, M. P., Cusimano, M., \& Stuss, D. T. (2007). Fast and efficient visuotemporal attention requires the cerebellum. Neuropsychologia 45, 3068-3074.

Segheir, M. L. (2008). Laterality index in functional MRI: Methodological issues. Magnetic Resonance Imaging 26, 594-601.

Sequeira, S. D. S., Specht, K., Hamalainen, H., \& Hugdahl, K. (2008). The effects of background noise on dichotic listening to consonantvowel syllables. Brain Language 107, 11-15.

Shivde, G., \& Thompson-Schill, H. L. (2004) Dissociating semantic and phonological maintenance using fMRI. Cognitive Affective and Behavioral Neuroscience 4, 10-19.

Smith, E. E., Gewa, A., Jonides, J., Miller, A., Reuter-Lorenz, P., \& Koeppe, R. A. (2000). The neural basis of task-switching in working memory: Effects of performance and aging. Proceedings of the National Academy of Sciences of the United States of America 98, 2095-2100.

Soderlund, G. B. W., Sikstrom, S., Loftesnes, J. M., Sonuga-Barke, E. J. (2010). The effects of background white noise on memory performance in inattentive school children. Behavioural and Brain Functions 6, 55.

Sohn, J., \& Lee, M. (2000). Selective attention system using new active noise controller. Neurocomputing 31, 197-204.

Sorqvist, P. (2010). Effects of aircraft noise and speech on prose memory: What role for working memory capacity? Journal of Environmental Psychology 30, 112-118.

Sun, X., Zhang, X., Chen, X., Zhang, P., Bao, M., Zhang, D., ... Hu, X. (2005). Age-dependent brain activation during forward and backward digit recall revealed by fMRI. NeuroImage 26, 36-47.

Sweet, L. H., Paskavitz, J. F., Haley, A. P., Gunstad, J. J., Mulligan, R. C., Nyalakanti, P. K., Cohan, R. A. (2008). Imaging phonological similarity effects on verbal working memory. Neuropsychologia $46,1114-1123$. 
Teder-Salejarvi, W. A., Pierce, K. L., Courchesne, E., Hillyard, S. A. (2005). Auditory spatial localization and attention deficits in autistic adults. Cognitive Brain Research 23, 221-234.

Toepper, M., Markowitsch, H. J., Gebhardt, H., Beblo, T., Thomas, C., Gallhofer, B., ... Sammer, G. (2010). Hippocampal involvement in working memory encoding of changing locations: An fMRI study. Brain Research 1354, 91-99.

Townsend, J., Westerfield, M., Leaver, E., Makeig, S., Jung, T., Pierce, K., \& Courchesne, E. (2001). Event-related brain response abnormalities in autism: evidence for impaired cerebello-frontal spatial attention networks. Cognitive Brain Research 11, 127-145.
Wong, P. C. M., Jin, J. X., Gunasekera, G. M., Abel, R., Lee, E. R., $\&$ Dhar, S. (2009). Aging and cortical mechanisms of speech perception in noise. Neuropsychologia 47, 693-703.

Yamamoto, Y., Hidaka, I., Nozaki, D., Iso-o, N., Soma, R., \& Kwak, S. (2002). Noise-induced sensitization of human brain. Physica A 314, 53-60.

Yusoff, S., \& Ishak, A. (2005). Evaluation of urban highway enviromental noise pollution. Sains Malaysiana 34(2), 81-87.

Zhou, X., Chen, C., Zhang, H., Xue, G., Dong, Q., Jin, Z., ... Chen, C. (2006). Neural substrates for forward and backward recitation of numbers and the alphabet: A close examination of the role of intraparietal sulcus and perisylvian areas. Brain Research 1099, 109-120. 